\title{
Depression and Sleep Quality among Iranian Women with Breast Cancer
}

\author{
Seyed Afshin Shorofi' ${ }^{1,2 *}$, Fereshteh Nozari-Mirarkolaei ${ }^{3}$, Paul Arbon $^{4}$, Masoumeh \\ Bagheri-Nesamie ${ }^{1}$
}

\begin{abstract}
Background and purpose: Breast cancer causes many psychological disorders such as sleep disturbances and depression. The current study was, therefore, intended to describe sleep quality and depression and to identify the association between these two psychological disorders among Iranian women with breast cancer. Materials and methods: This descriptive, analytical, cross-sectional study was carried out on 120 women with non-metastatic unilateral breast cancer undergoing chemotherapy in an outpatient chemotherapy unit of a major public hospital. A total of 120 women who had already undergone mastectomy procedure were selected via convenience sampling method. Data were collected by the Beck Depression Inventory-II (BDI-II) and the Pittsburgh Sleep Quality Index (PSQI). Results: The mean score on BDI-II was $13.40( \pm 6.51)$, and $30 \%(n=36)$ of women had mild depression and $14.2 \%(n=17)$ reported moderate-to-severe depression. The mean global score of sleep quality was found to be $6.48( \pm 2.62)$. Furthermore, $50.8 \%$ $(n=61)$ of women obtained a global PSQI score of 5. A positive correlation was found between depression scores and sleep quality scores $(\mathrm{p}=0.001, \mathrm{r}=0.48)$. Depression was also correlated with age, number of children, household gross income, sleep duration, sleep latency, and type of mastectomy procedure $(\mathrm{p}<0.05)$. Moreover, subjective sleep quality was correlated with number of chemotherapy sessions $(\mathrm{p}=0.001, \mathrm{r}=-0.67)$ and daytime dysfunction $(\mathrm{p}=0.001, \mathrm{r}=0.78)$. A positive correlation was also observed between sleep disturbances and habitual sleep efficiency $(\mathrm{p}=0.02, \mathrm{r}=0.65)$. Conclusion: In conclusion, 30\% of women had mild depression and 14.2\% reported moderate-to-severe depression. The mean global score of sleep quality was found to be $6.48( \pm 2.62)$, suggesting poor sleep quality. Furthermore, over half of the participants $(50.8 \%)$ obtained a global PSQI score of 5 or greater which is indicative of poor sleep quality. A positive moderate correlation was also observed between depression and poor sleep quality.
\end{abstract}

Keywords: Breast cancer- depression- sleep quality- BDI- PSQI

Asian Pac J Cancer Prev, 22 (11), 3433-3440

\section{Introduction}

Breast cancer is the most common type of cancer in women with the highest mortality rate at the age of 40-44 years (Bray et al., 2018). There is an estimated 2.1 million new cases of breast cancer each year. In 2018, about 627000 women died from this cause worldwide, accounting for almost $15 \%$ of all cancer deaths among women (WHO, 2019). The fifth most common cause of cancer death, breast cancer is also the most frequent cancer in Iranian women, estimated to comprise $24.4 \%$ of all cancers. About 6160 new cases of breast cancer are diagnosed in women each year in Iran (Farhood et al., 2018).

Breast cancer causes many problems for the patient. Psychological disorders such as sleep disturbances
(Lowery-Allison et al., 2018), depression, anxiety (Park et al., 2018), helplessness, social withdrawal and stress (Bener et al., 2017) are some of these problems. Depression is one of the most common comorbidities of cancer patients (Mausbach et al., 2018). It seems that several factors are associated with depression in women with breast cancer, including age, educational attainment, lymphedema, self-esteem levels, body image (Boing et al., 2019), type of breast surgery (Boing et al., 2019; Rady et al., 2018), employment status, household income, obesity, marital status, side effects of chemotherapy on fertility, sexual function and perimenopausal period (Huang et al., 2019). Moreover, there is a significant association between depression and sleep disorder (Mansano-Schlosser et al., 2017).

One of the most common complaints in patients

${ }^{1}$ Traditional and Complementary Medicine Research Center, Addiction Institute, Mazandaran University of Medical Sciences, Sari, Iran. ${ }^{2}$ Adjunct Research Fellow, Flinders University, Adelaide, Australia. ${ }^{3}$ Student Research Committee, School of Nursing and Midwifery, Mazandaran University of Medical Sciences, Sari, Iran. ${ }^{4}$ Torrens Resilience Institute, Flinders University, Adelaide, Australia; School of Nursing and Health Sciences, Flinders University, Adelaide, Australia. *For Correspondence: ashorofi@yahoo.com (SA. Shorofi) 
with cancer is reported to be sleep disorders observed in about $50 \%$ of these patients, and most frequently in women and patients with breast cancer (Izci et al., 2016). Patients diagnosed with breast cancer report higher rates of sleep disorders (67\% to $90 \%)$ compared with other cancer patients. In fact, these patients are two times more likely to report sleep problems than women free from cancer, and these problems may continue up to 10 years post-treatment. Sleep problems may lead to substantial adverse consequences, including "poor healthrelated quality of life, fatigue, poor healing, cognitive dysfunction, lost work productivity, safety issues (e.g., accidents), poor relationships, and increased health care costs" (Otte et al., 2016).

Sleep disorders and depression commonly occur together in breast cancer survivors and interact with each other to negatively affect patients' quality of life (Fiorentino et al., 2011). Sleep disorders, such as hypersomnia, short sleep duration, restless legs syndrome, parasomnia, sleep apnea and insomnia, experienced by breast cancer survivors were detected to be associated with decreased physical function and emotional states (including, depressive symptoms and distress), while low sleep quality was observed to be associated with greater levels of anxiety and depression (Cho and Hwang, 2021). However, "sparse population-based data exist about the contribution of insomnia symptoms to occurrence of depressive symptoms in breast cancer survivors" (Haque et al., 2020). A study by Ho et al., (2015) found a correlation between depression, fatigue and sleep quality in both premenopausal and postmenopausal women with breast cancer. Difficulties in falling asleep and waking up in the middle of the night have been found to be significantly associated with greater depression in breast cancer patients (Mansano-Schlosser et el., 2017). Depressive symptoms are also the identified predictors of subjective sleep disturbance in women diagnosed with breast cancer before, during and after radiation therapy (Dhruva et al., 2012). According to Ancoli-Israel et al., (2014), breast cancer patients had more severe symptoms than those without cancer even before undergoing chemotherapy. Women with breast cancer had significantly worse fatigue, more disrupted circadian activity rhythms, worse sleep and more depression symptoms at the end of four cycles of chemotherapy compared with their own baseline levels and cancer-free controls. Although the symptoms returned to baseline levels by one year, they were still worse than those in cancer-free controls.

It is important to point out that both depression and sleep disorders are significantly associated with an increase of mortality rate (Bach et al., 2020). An association has been found between mild sleep disturbance and increased mortality in premenopausal women with breast cancer (Vaughn et al., 2018). Depression can also bring about a lower quality of life and seriously compromise patient outcomes, resulting in higher rates of mortality in cancer (Mansano-Schlosser et al., 2017). A systematic review and meta-analysis presented reasonable evidence that psychological distress (symptoms of depression and anxiety) is related to an increased mortality rate and poorer survival in patients with cancer (Wang et al., 2020).
According to the available databases, no study has been conducted in Iran to date on depression and sleep quality in women with breast cancer undergoing chemotherapy. The cultural differences in manifestations of depression (Falicov, 2018), different strategies used to cope with breast cancer (Sajadian et al., 2017; Kvillemo and Bränström, 2014), and the relevance of sleep-related behaviours to social and cultural factors (Grandner et al., 2013) may affect the outcomes of various studies conducted for the assessment of depression and sleep quality in patients with cancer. The current study was, therefore, intended to describe sleep quality and depression and to identify the association between these two psychological disorders among Iranian women with breast cancer.

\section{Materials and Methods}

\section{Sample and sampling method}

This descriptive, analytical, cross-sectional study was conducted from December 2018 to September 2019. The study population consisted of women with breast cancer undergoing chemotherapy in an outpatient chemotherapy unit of a major public hospital affiliated to Mazandaran University of Medical Sciences (Sari, Iran). Eligibility criteria included women diagnosed with non-metastatic unilateral breast cancer aged $\geq 18$ who had undergone mastectomy (any type) and were being treated with chemotherapy with no experience of stressful events (other than breast removal operation; e.g. death of a beloved one) within the last six months (Zwielewski and Sant'Ana, 2019), no history of depression or other mental disorders, and no history of taking sleeping medication and tranquillizers prior to the diagnosis of breast cancer. We needed a sample size of 120 , which was based on a previous study (Musarezaie et al., 2014) and power analysis calculation. Participants were selected via convenience sampling method due to the relatively small population of patients with breast cancer.

\section{Measurement instruments}

Data were collected using a socio-demographic/ disease-related information form, the Pittsburgh Sleep Quality Index (PSQI), and the Beck Depression InventoryII (BDI-II). The socio-demographic/disease-related information form collected data on age; number of children; native or non-native to Mazandaran province; marital status; educational attainment; occupation; household gross income; emotional support resources; side of involved breast; type of mastectomy procedure; time elapsed since mastectomy; number of chemotherapy sessions received; history of depression, sleep disturbance, hospital admission; family history of breast and other cancers; and history of taking sleeping medication.

A commonly used indicator of the severity of depression, the BDI-II is a self-report inventory consisting of 21 sets of statements, each ranked on the basis of severity and scored on a scale value of 0 to 3 . The total score ranges from 0 to 63 , with higher scores indicating greater severity of depression. Scores of 0-13 indicate minimal depression, 14-19 mild depression, 20-28 moderate depression, and 29-63 severe depression 
(Smarr and Smarr, 2011). The Persian version of BDI-II has demonstrated acceptable psychometric properties of validity and reliability in different populations (Hamidi et al., 2015; Ghassemzadeh et al., 2005; Ahmadi et al., 2019; Rahimi, 2014).

The PSQI, developed by Buysse et al., (1989), is a valid and reliable instrument for the assessment of sleep quality and disturbances in the previous month. The PSQI has demonstrated a high degree of internal consistency with Cronbach's [alpha] coefficient of 0.83 and validity of 0.75 . In addition, the Persian version of PSQI has also been reported to have optimal validity and reliability in different populations (Farrahi Moghaddam et al., 2012; Mohammad Gholi Mezerji et al., 2017; Nazifi et al., 2014). The PSQI consists of 19 self-rated questions which evaluates 7 sleep components including subjective sleep quality, sleep latency, sleep duration, habitual sleep efficiency, sleep disturbances, use of sleeping medication, and daytime dysfunction. Each component is weighted equally on a 0-3 scale, with a global score ranging from 0 to 21. A global score of 5 or greater is indicative of poor sleep quality (Buysse et al., 1989).

\section{Procedures}

Participants were selected by convenience sampling method and recruited to the study. Socio-demographic/ disease-related characteristics were collected from participants through face-to-face interviews and patients' medical files. Depression was measured using the BDI-II and sleep quality was evaluated with the PSQI. All data collection tools were completed in the presence of the researcher.

\section{Data analysis}

The collected data were coded and statistically analyzed with SPSS 22.0 software (SPSS, Inc., Chicago, IL) using descriptive statistics (mean, percentage and standard deviation) and analytical tests (Chi-square and
Pearson's correlation coefficient). The significance level was set at a $\mathrm{p}$ value of $<0.05$.

\section{Ethical considerations}

The study protocol was approved by the Bioethics Committee of Mazandaran University of Medical Sciences (MAZUMS.IR.S.261) in accordance with the Helsinki Declaration in its latest version. Additional access approvals were obtained from the participating hospital. Our participants were initially properly informed about study aims, procedures, and confidentiality. They were also assured that they would be free to withdraw from the study at any time without giving a reason and without any adverse effect on their continued care. All participants signed an informed consent form in which the study procedures and their rights had been delineated.

\section{Results}

Sociodemographic and disease-related characteristics

A total of 120 patients who met the selection criteria participated in the study with a mean age of 45.7 $( \pm 4.52)$ years (range: $29-63$ years). Less than half of the participants $(\mathrm{n}=56,46.7 \%)$ stated that they were in their menopausal period. In addition, $45.8 \%$ of women had a family history of cancer. The average time elapsed since mastectomy was $5.52( \pm 3.5)$ months. Patients' socio-demographic/disease-related characteristics are summarized in Table 1.

\section{Levels of sleep and depression}

The mean score on BDI-II was $13.40( \pm 6.51)$. Approximately one third of women $(n=36,30 \%)$ had mild depression and $14.2 \%(n=17)$ reported moderate-to-severe depression (Table 2). The mean global score of sleep quality was also found to be $6.48( \pm 2.62)$. Furthermore, over half of the participants $(50.8 \%, n=61)$ obtained a global PSQI score of 5 or greater which is indicative of

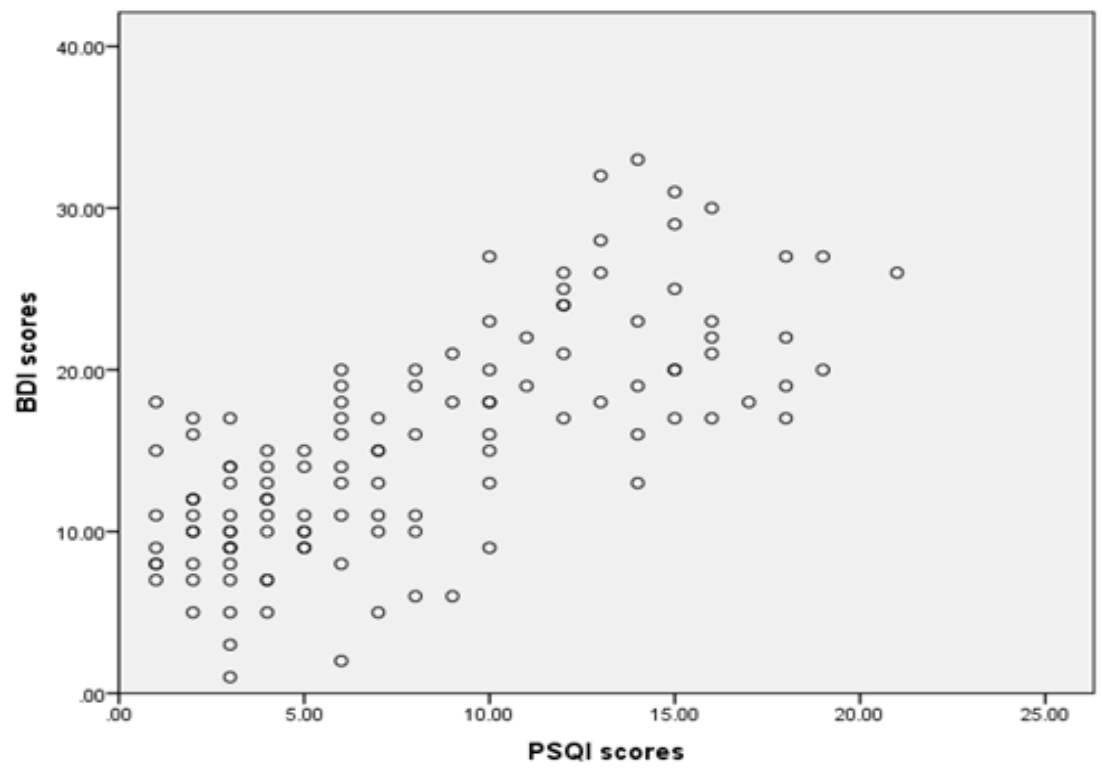

Figure 1. The Scatter Graph Showing a Positive Moderate Correlation between PSQI Scores and BDI Scores. BDI scores, Beck Depression Inventory scores; PSQI scores, Pittsburgh Sleep Quality Index scores 
Table 1. Socio-Demographic/ Disease-Related Characteristics of the Participants $(n=120)$

\begin{tabular}{|c|c|c|c|}
\hline Characteristics & & Characteristics & \\
\hline \multirow[t]{3}{*}{ Age } & (Years, $\mathrm{M} \pm \mathrm{SD}$ ) & Menopausal symptoms & $\mathrm{n}(\%)$ \\
\hline & $45.7( \pm 4.52)$ & Yes & $56(46.7)$ \\
\hline & & No & $64(53.3)$ \\
\hline Native of Mazandaran province & $\mathrm{n}(\%)$ & & \\
\hline Yes & $114(95)$ & Emotional support resources & $\mathrm{n}(\%)$ \\
\hline \multirow[t]{2}{*}{ No } & $6(5)$ & Spouse & $52(43.3)$ \\
\hline & & Parents & $24(20)$ \\
\hline Number of children & $\mathrm{n}(\%)$ & Children & $33(27.5)$ \\
\hline 0 & $4(3.3)$ & Other & $11(9.1)$ \\
\hline 1 & $11(9.2)$ & Family history of cancer & $\mathrm{n}(\%)$ \\
\hline 2 & $47(39.2)$ & Yes & $55(45.8)$ \\
\hline 3 & $45(37.5)$ & No & $65(54.2)$ \\
\hline 4 & $4(8.3)$ & & \\
\hline 5 & $2(1.7)$ & Family history of breast cancer & $\mathrm{n}(\%)$ \\
\hline \multirow[t]{2}{*}{6} & $1(0.8)$ & Yes & $48(23.3)$ \\
\hline & & No & $92(76.7)$ \\
\hline Marital status & $\mathrm{n}(\%)$ & & \\
\hline Single & $2(1.7)$ & History of hospital admission & $\mathrm{n}(\%)$ \\
\hline Married & $109(90.8)$ & Yes & $46(38.3)$ \\
\hline Divorced/separated & $3(2.5)$ & No & $74(61.7)$ \\
\hline \multirow[t]{2}{*}{ Widow } & $6(5)$ & & \\
\hline & & Involved breast & $\mathrm{n}(\%)$ \\
\hline Educational attainment & $\mathrm{n}(\%)$ & Right & $62(51.7)$ \\
\hline Academic degree & $33(27.5)$ & Left & $58(48.3)$ \\
\hline \multirow[t]{2}{*}{ Diploma and lower } & $87(72.5)$ & & \\
\hline & & Type of mastectomy procedure & $\mathrm{n}(\%)$ \\
\hline Employment & $\mathrm{n}(\%)$ & Partial & $75(62.5)$ \\
\hline Housewife & $96(80)$ & Radical & $9(7.5)$ \\
\hline Employee & $6(5)$ & Total & $36(30)$ \\
\hline Self-employed & $15(12.5)$ & & \\
\hline Student & $1(0.8)$ & Time elapsed since mastectomy & (years, $\mathrm{M} \pm \mathrm{SD}$ ) \\
\hline Unemployed & $1(0.8)$ & & $5.52( \pm 3.5)$ \\
\hline \multirow[t]{2}{*}{ Retired } & $1(0.8)$ & Number of chemotherapy sessions & $\mathrm{n}(\%)$ \\
\hline & & $<5$ & $66(55)$ \\
\hline Household gross income & $\mathrm{n}(\%)$ & $>5$ & $54(45)$ \\
\hline Good & $6(5)$ & & \\
\hline Moderate & $22(18.3)$ & & \\
\hline Low & $92(76.7)$ & & \\
\hline
\end{tabular}

$\mathrm{M} \pm \mathrm{SD}:$ mean \pm standard deviation; $\mathrm{n}(\%)$ : number (percent).

poor sleep quality. Table 3 depicts the mean ( \pm standard deviation) scores on global PSQI and PSQI subscales.

Negative correlations were also found between depression and age $(p=0.002, r=-0.72)$, number of children $(\mathrm{p}=0.02, \mathrm{r}=-0.54)$, and household gross income $(p=0.003, r=-0.65)$. As such, younger patients, those with fewer children and patients with lower household gross income were more likely to experience higher levels of depression. In addition, the Chi-square test indicated that there was a significant relationship between depression and type of mastectomy procedure $(\mathrm{p}=0.04)$ so that women with total and radical mastectomy were more likely to report higher levels of depression than the others. The Pearson's correlation coefficient showed that there was a significant negative correlation between number of chemotherapy sessions and subjective sleep quality in breast cancer patients $(\mathrm{p}=0.001, \mathrm{r}=-0.67)$. Moreover, subjective sleep quality was positively significantly correlated with daytime dysfunction $(\mathrm{p}=0.001, \mathrm{r}=0.78)$. A positive correlation was also observed between sleep disturbances and habitual sleep efficiency $(p=0.02$, $\mathrm{r}=0.65)$. 
Table 2. Depression Scores in Women with Breast Cancer

\begin{tabular}{llccc}
\hline BDI scores & Level of depression & $\mathrm{n}(\%)$ & Range & Mean $( \pm$ standard deviation $)$ \\
\hline $0-13$ & Minimal depression & $67(55.8)$ & $2-12$ & \\
$14-19$ & Mild depression & $36(30)$ & $14-19$ & $13.40( \pm 6.51)$ \\
$20-28$ & Moderate depression & $15(12.5)$ & $20-26$ & \\
$29-63$ & Severe depression & $2(1.7)$ & $29-38$ & \\
\hline
\end{tabular}

BDI-score, Beck Depression Inventory-score

Table 3. Mean ( \pm standard deviation) Scores on Global PSQI and PSQI Subscales

\begin{tabular}{lcc}
\hline Subscales & $\begin{array}{c}\text { Mean } \\
( \pm \text { standard deviation })\end{array}$ & Range \\
\hline Subjective sleep quality & $1.34( \pm 0.9)$ & $0-2$ \\
Sleep latency & $1.07( \pm 0.3)$ & $0-3$ \\
Sleep duration & $0.74( \pm 0.4)$ & $1-3$ \\
Habitual sleep efficiency & $2.16( \pm 1.5)$ & $1-3$ \\
Sleep disturbances & $1.47( \pm 0.6)$ & $0-3$ \\
Use of sleeping medication & $0.40( \pm 0.2)$ & $1-2$ \\
Daytime dysfunction & $0.85( \pm 0.7)$ & $0-2$ \\
Global PSQI & $6.48( \pm 2.62)$ & $2-15$ \\
\hline
\end{tabular}

PSQI, Pittsburgh Sleep Quality Index

\section{Association between sleep quality and depression}

The Pearson's correlation coefficient showed a positive moderate correlation between depression scores and sleep quality scores ( $\mathrm{p}=0.001, \mathrm{r}=0.48$ ) (Figure 1). Furthermore, depression was positively correlated with sleep duration $(\mathrm{p}=0.01, \mathrm{r}=0.58)$ and sleep latency $(\mathrm{p}=0.02, \mathrm{r}=0.49)$.

\section{Discussion}

This study examined the relationship between depression and sleep quality in Iranian women with breast cancer. Nearly half the participants $(44.2 \%)$ reported having mild-to-severe depression, which is considerably higher than the prevalence of depression among Iranian women reported by Musarezaie et al., (2015) and Noorbala et al., (2017). The prevalence of depression among women with breast cancer was reported in 2017 to be $44 \%$ in the USA (Park et al., 2018). It is reasonable to assume that differences in socioeconomic status including age, educational attainment (Boing et al., 2019; Freeman et al., 2016), income level (Huang et al., 2019; Freeman et al., 2016) and marital status (Rady et al., 2018; Huang et al. 2019, Freeman et al., 2016); religiosity and religious coping (Ng et al., 2017); coping patterns (Li et al., 2017); menopausal state; family history of breast cancer; breast cancer staging; type of breast surgery (Boing et al., 2019; Rady et al., 2018); diagnosis of other diseases; lymphedema; self-esteem; body image (Boing et al., 2019); obesity; and side effects of chemotherapy on fertility and sexual function (Huang et al., 2019) may explain inconsistencies in the reported prevalence of depression among women with breast cancer by different studies. Depression may itself bring about many negative consequences, including longer hospital stays, increased physical distress, poorer adherence to treatment, lower quality of life, and high desire for hastened death ( $\mathrm{Li}$ et al., 2016). Therefore, various and effective strategies (such as psychosocial interventions) are required to prevent and treat co-morbid depression and poor mental health among women with breast cancer. Psycho-oncologists can play a critical role in the screening, assessment and management of depression in this group of patients. They may use combined approaches (including psychotherapy and psychopharmacology) to manage, prevent and reduce the distress and psychosocial morbidity associated with cancer. Other healthcare professionals, particularly oncologists, nurses and social workers, can also provide patients and their families with psychological, social and emotional support, and help them cope with the demands of treatment and the uncertainty over the course of treatment and prognosis, and improve their quality of life.

Our results also demonstrated a relationship between depression and type of mastectomy procedure so that women with total and radical mastectomy were more likely to report higher levels of depression than their counterparts with partial mastectomy. Boing et al. (2019), for example, concluded that breast surgery, specifically radical mastectomy, was associated with depression symptoms. A systematic review and meta-analysis also found no significant differences among three types of surgery (total mastectomy, breast conserving therapy, and breast reconstruction) on depression in female breast cancer patients (Zhang et al., 2018). Notwithstanding a relationship between type of mastectomy procedure and depression in our study, it is still difficult to conclusively determine that our participants' depression was caused by cancer or mastectomy (Kim et al., 2017).

Younger patients, those with fewer children and patients with lower income in our study were more likely to experience higher levels of depression. It is possible that younger breast cancer women experience more disruption in body image, anxiety, sleep disorders, dissatisfaction with relationships, fear of relapse (Mendoza et al., 2017), worse sexual problems (Mendoza et al., 2017; Jankowska, 2013), higher rates of depression, poorer quality of life and psychological well-being than older counterparts (Jankowska, 2013). They may also have relatively higher concerns about loss of fertility and premature menopause (Jankowska, 2013). It is possible that women with fewer children in the present study were receiving less support than women with more children, which could have a negative impact on their mental health. Children could be an important source of support for these highly stressed women. Christie et al., (2010) found that single breast cancer women, who were considered to feel lonely and 
deprived of emotional support, reported higher depressive symptoms. Our finding that women with lower income were more likely to have higher depression than those with higher income is in line with the extant literature (Chen et al., 2009; Srivastava et al., 2016; Wei et al., 2019).

The findings of the present study indicate that over half of the women with breast cancer $(50.8 \%)$ had poor sleep quality. The prevalence of poor sleep quality in breast cancer patients varies greatly, depending on study design, sleep disturbance scale used, different clinical and demographic profiles of samples studied, and type of cancer treatment received by women (Khorami et al., 2012; Berger et al., 2019; McManus et al., 2019; Alifiyanti et al., 2018; de Araújo et al., 2014; Carroll et al., 2019; Vin-Raviv et al., 2018; Gonzalez and Lu, 2018). We also found a positive moderate correlation between depression and poor sleep quality. There is a complex relationship between sleep disturbance and mood state because sleep disturbance can result in such psychological disorders as depression, anxiety and emotional changes, and these conditions can in turn interfere with sleep (SleepFoundation.org, updated 18 September 2020). Sleep disturbances and depression which can co-occur as part of symptom clusters in these patients (Ho et al., 2015) can be lasting and have a negative impact on patients' quality of life. Therefore, effective management of sleep disturbances and psychological disorders in women with breast cancer is of great importance.

This study has several limitations. The current study was conducted on post-mastectomy women with non-metastatic unilateral breast cancer undergoing chemotherapy in an outpatient chemotherapy unit of a major public hospital affiliated to Mazandaran University of Medical Sciences (Sari, Iran), which may limit the generalizability of the findings. Another potential limitation of this study pertains to a relatively small sample size of patients, although it is important to consider that the adequacy of the sample size was ensured by power analysis calculation. Studies with larger sample sizes would certainly impart more power to analyze the various variables reported. Another potential limitation of the present study is that the findings are based on data from Iranian women with low socioeconomic status which may limit the generalizability of the data. The final limitation is the use of self-report measures which may have not reflected participants' actual sleep quality and depression.

Several insights were obtained from this study. Effective management of sleep disturbances and depression should be one of our clinical priorities for women with breast cancer. More attention should also be paid to depression and sleep disturbances by healthcare professionals, particularly nurses and physicians, providing care and medical treatment for women with breast cancer. In addition, oncology social workers have an important role in helping these patients with practical needs, like finding sources and financial assistance. It is also worth noting that coping strategies adopted by Iranian cancer patients mainly stem from their religious beliefs that should be respected by healthcare professionals as spiritual and religious beliefs have protective effects against depression (Mahdanian, 2018).
In conclusion, $30 \%$ of women had mild depression and $14.2 \%$ reported moderate-to-severe depression. The mean global score of sleep quality was found to be $6.48( \pm 2.62)$, suggesting poor sleep quality. Furthermore, over half of the participants $(50.8 \%)$, obtained a global PSQI score of 5 or greater which is indicative of poor sleep quality. In addition, a positive moderate correlation was observed between depression and poor sleep quality. Depression and sleep quality were also associated with diverse socio-demographic factors. One area for future research is to explore the relationship between coping with cancer diagnosis, depression and sleep quality among women with breast cancer. Future research should also incorporate both subjective and objective measures of sleep (such as polysomnography and actigraphy plus physician/nurseor patient-administered tools) and depression (such as laboratory methods plus physician/nurse- or patientadministered tools) in breast cancer survivors.

\section{Author Contribution Statement}

SA. Shorofi: Conceptualization; Data Curation (supporting); Funding Acquisition (equal); Investigation (equal); Methodology; Project Administration; Supervision; Validation; Visualization; Writing Original Draft (lead); Writing - Review \& Editing. F. Nozari: Formal Analysis (equal), Funding Acquisition (equal), Investigation (equal), Writing - Original Draft (supporting), Data Curation (lead). P. Arbon: Writing - Original Draft. M. Bagheri-Nesami: Formal Analysis (equal).

\section{Acknowledgements}

The study protocol was approved by the Bioethics Committee of Mazandaran University of Medical Sciences (MAZUMS.IR.S.261). The authors thank all the patients who participated in the study.

\section{Funding statement}

This study was funded by Mazandaran University of Medical Sciences, Sari, Iran.

\section{Conflict of interest}

The authors declare no conflict of interest.

\section{References}

Ahmadi SM, Masjediarani A, Bakhtiari M, Davazdahemamy MH, Mohamadian R (2019) Psychometric properties of Persian version of Beck Depression Inventory in coronary patients. Univ Med, 38, 33-40.

Alifiyanti D , Hermayanti Y, Setyorini D (2018). The sleep quality of breast cancer patients. $J N C, \mathbf{1}$, doi : https://doi. org/10.24198/jnc.v1i2.16140.

Ancoli-Israel S, Liu L, Rissling M, et al (2014). Sleep, fatigue, depression, and circadian activity rhythms in women with breast cancer before and after treatment: a 1-year longitudinal study. SCC, 22, 2535-45.

Bach L, Kalder M, Kostev K (2020). Depression and sleep disorders are associated with early mortality in women with breast cancer in the United Kingdom. J Psychiatr Res, 
In Press. https://doi.org/10.1016/j.jpsychires.2020.11.036.

Bener A, Alsulaiman R, Doodson L, Agathangelou T (2017). Depression, hopelessness and social support among breast cancer patients. Asian Pac J Cancer Prev, 18, 1889-96.

Berger AM, Kupzyk KA, Djalilova DM, Cowan KH (2019). Breast cancer collaborative registry informs understanding of factors predicting sleep quality. SCC, 27, 1365-73.

Boing L, Pereira GS, Araújo CD, et al (2019). Factors associated with depression symptoms in women after breast cancer. Rev Saúde Pública, 53, doi: 10.11606/s15188787.2019053000786.

Bray F, Ferlay J, Soerjomataram I, et al (2018). Global cancer statistics 2018: GLOBOCAN estimates of incidence and mortality worldwide for 36 cancers in 185 countries. $C A$ Cancer J Clin, 68, 394-424.

Buysse DJ, Reynolds CF, Monk TH, Berman SR, Kupfer DJ (1989). The Pittsburgh Sleep Quality Index: a new instrument for psychiatric practice and research. Psychiatry Res, 28, 193-213.

Carroll JE, Small BJ, Tometich DB, et al (2019). Sleep disturbance and neurocognitive outcomes in older patients with breast cancer: Interaction with genotype. Cancer, 125, 4516-24.

Chen X, Zheng Y, Zheng W, et al (2009). Prevalence of depression and its related factors among Chinese women with breast cancer. Acta Oncol, 48, 1128-36.

Cho OH, Hwang KH (2021). Association between sleep quality, anxiety and depression among Korean breast cancer survivors. Nurs Open, 8, 1030-37.

Christie KM, Meyerowitz BE, Maly RC (2010). Depression and sexual adjustment following breast cancer in low-income Hispanic and non-Hispanic White women. Psychooncology, 19, 1069-77.

de Araújo DV, Costa RHS, Justino DCP, et al (2014). Evaluation of sleep quality in patients with breast cancer. J Sleep Disor Treat Care, 3, doi:10.4172/2325-9639.1000142.

Dhruva A, Paul SM, Cooper BA, et al (2012). A longitudinal study of measures of objective and subjective sleep disturbance in patients with breast cancer before, during, and after radiation therapy. J Pain Symptom Manage, 44, 215-28.

Falicov CJ (2018). Culture, society and gender in depression. J Fam Ther, 25, 371-87.

Farhood B, Geraily G, Alizadeh A (2018). Incidence and mortality of various cancers in Iran and compare to other countries: A review article. Iran J Public Health, 47, 309-16.

Farrahi Moghaddam J, Nakhaee N, Sheibani V, Behshid Garrusi B, Amirkafi A (2012). Reliability and validity of the Persian version of the Pittsburgh Sleep Quality Index (PSQI-P). Sleep Breath, 16, 79-82.

Fiorentino L, Rissling M, Liu L, Ancoli-Israel S (2011). The symptom cluster of sleep, fatigue and depressive symptoms in breast cancer patients: Severity of the problem and treatment options. Drug Discov Today Dis Models, 8, 167-73.

Freeman A, Tyrovolas S, Koyanagi A, et al (2016). The role of socio-economic status in depression: results from the COURAGE (aging survey in Europe). BMC Public Health, 16, doi.org/10.1186/s12889-016-3638-0.

Ghassemzadeh H, Mojtabai R, Karamghadiri N, Ebrahimkhani N (2005). Psychometric properties of a Persian-language version of the Beck Depression Inventory - Second edition: BDI-II-Persian. Depress Anxiety, 21, 185-92.

Gonzalez BD, Lu Q (2018). Sleep disturbance among Chinese breast cancer survivors living in the USA. SCC, 26, 1695-98.

Grandner MA, Patel NP, Jean-Louis G, et al (2013). Sleep-related behaviors and beliefs associated with race/ ethnicity in women. Natl Med Assoc, 105, 4-15.
Hamidi R, Fekrizadeh Z, Azadbakht M, et al (2015). Validity and reliability Beck Depression Inventory-II among the Iranian elderly population. J Sabzevar Univ Med Sci, 22, 189-98.

Haque R, Hsu JW, Avila C, et al (2020). Insomnia and susceptibility to depressive symptoms and fatigue in diverse breast cancer survivors. J Womens Health, 00, DOI: 10.1089/ jwh.2019.8135.

Ho SY, Rohan KJ, Parent J, Tager FA, Mckinley PS (2015). A longitudinal study of depression, fatigue, and sleep disturbances as a symptom cluster in women with breast cancer. J Pain Symptom Manage, 49, 707-15.

Huang Z, Zhao J, Ding K, et al (2019). Depression involved in self-reported prospective memory problems in survivors of breast cancer who have received chemotherapy. Medicine (Baltimore), 98, doi: 10.1097/md.0000000000015301.

Izci F, İlgün AS, Fındıklı F, Özmen V (2016). Psychiatric symptoms and psychosocial problems in patients with breast cancer. J Breast Health, 12, 94-101.

Jankowska M (2013). Sexual functioning in young women in the context of breast cancer treatment. Rep Pract Oncol Radiother, 18(4), 193-200.

Khorami Rad A, Noroozi M, Ahmari Tehran H, Rahmani A (2012). Quality of sleep and related factors in breast cancer patients receiving chemotherapy in Qom 2011. Iran J Breast Dis, 4, 51-60.

Kim MS, Kim SY, Kim JH, Park B, Choi HG (2017). Depression in breast cancer patients who have undergone mastectomy: A national cohort study. PLoS One, 12, doi: org/10.1371/ journal.pone. 0175395 .

Kvillemo P, Bränström R (2014). Coping with breast cancer: a meta-analysis. PLoS One, 9, doi.org/10.1371/journal. pone. 0112733 .

Li L, Li S, Wang Y, et al (2017). Coping profiles differentiate psychological adjustment in Chinese women newly diagnosed with breast cancer. Integr Cancer Ther, 16, 196-204.

Li M, Kennedy EB, Byrne N, et al (2016). Management of depression in patients with cancer: A clinical practice guideline. JCO Oncol Pract, 12, 747-56.

Lowery-Allison AE, Passik SD, Cribbet MR, et al (2018). Sleep problems in breast cancer survivors: 1-10 years posttreatment. Palliative Supportive Care, 16, 325-34.

Mahdanian A (2018). Religion and Depression: A Review of the Literature. J Psychiatry Behav Health Forecast, 1, 1003.

Mansano-Schlosser TC, Ceolim MF, Valerio TD (2017). Poor sleep quality, depression and hope before breast cancer surgery. Appl Nurs Res, 34, 7-11.

Mausbach BT, Bos T, Irwin SA (2018). Mental health treatment dose and annual healthcare costs in patients with cancer and major depressive disorder. Health Psychol, 37, 1035-40.

McManus S, Zaleta AK, Miller MF, et al (2019). Sleep disturbance and quality of life among breast cancer survivors. Cancer Res, 79, 50-62.

Mendoza N, Molero F, Criado F, Cornellana MJ, González E (2017). Sexual health after breast cancer: Recommendations from the Spanish menopause society, Federación Española de Sociedades de Sexología, Sociedad Española de Médicos de Atención Primaria and Sociedad Española de Oncología Médica. Maturitas, 105, 126-31.

Mohammad Gholi Mezerji N, Naseri P, Omraninezhad Z, Shayan Z (2017). The reliability and validity of the Persian version of Pittsburgh Sleep Quality Index in Iranian people. Avicenna J Neuropsychophysiol, 4, 95-102.

Musarezaie A, Momeni-Ghaleghasemi T, Gorji M (2015). Survey the anxiety and depression among breast cancer patients referred to the specialized Isfahan hospital of cancer, Iran. HSR, 10, 39-48. 
Nazifi M, Mokarami H, Akbaritabar A, Kalte HO, Rahi A (2014). Psychometric properties of the Persian translation of Pittsburgh Sleep Quality Index. Health Scope, 3, doi: $10.17795 /$ jhealthscope-15547.

Ng GC, Mohamed S, Sulaiman AH, Zainal NZ (2017). Anxiety and depression in cancer patients: the association with religiosity and religious coping. J Relig Health, 56, 575-90.

Noorbala AA, Bagheri Yazdi SA, Faghihzadeh S, et al (2017). A survey on mental health status of adult population aged 15 and above in the province of Ardebil, Iran. Arch Iran Med, 20, $11-4$.

Noorbala AA, Bagheri Yazdi SA, Faghihzadeh S, et al (2017). A survey on mental health status of adult population aged 15 and above in the province of Markazi, Iran. Arch Iran Med, 20, $79-82$.

Noorbala AA, Bagheri Yazdi SA, Faghihzadeh S, et al (2017). A survey on mental health status of adult population aged 15 and above in the province of Kermanshah, Iran. Arch Iran Med, 20, 59-62.

Noorbala AA, Bagheri Yazdi SA, Faghihzadeh S, et al (2017). A survey on mental health status of adult population aged 15 and above in the province of Ardebil, Iran. Arch Iran Med, 20, $11-4$.

Noorbala AA, Bagheri Yazdi SA, Faghihzadeh S, et al (2017). A survey on mental health status of adult population aged 15 and above in the province of Mazandaran, Iran. Arch Iran Med, 20, 83 -6.

Otte JL, Davis L, Carpenter JS, et al (2016). Sleep disorders in breast cancer survivors. SCC, 24, 4197-205.

Park EM, Gelber S, Rosenberg SM, et al (2018). Anxiety and depression in young women with metastatic breast cancer: A cross-sectional study. Psychosomatics, 59, 251-8.

Rady A, Elkholy O, Abouelwafa H, et al (2018). Demographic and clinical correlates of breast cancer patients with depression. Neuropsychiatry, 8, doi: 10.4172/Neuropsychiatry.1000476.

Rahimi C (2014). Application of the beck depression inventoryII in Iranian university students. Clin Psy Personality, 2, 173-88.

Sajadian A, RajiLahiji M, Motaharinasab A, Kazemnejad A, Haghighat S (2017). Breast cancer coping strategies after diagnosis: A six-month follow-up. $M C I, 1,12-6$.

Smarr KL, Keefer AL (2011). Measures of depression and depressive symptoms: Beck Depression Inventory-II (BDIII), Center for Epidemiologic Studies Depression Scale (CES-D), Geriatric Depression Scale (GDS), Hospital Anxiety and Depression Scale (HADS), and Patient Health Questionnaire-9 (PHQ-9). Arthritis Care Res, 63, 454-66.

Srivastava V, Ansari MA, Kumar A, et al (2016). Study of anxiety and depression among breast cancer patients from north India. Clin Psychiatry, 2, doi: 10.21767/2471-9854.100017.

The complex relationship between sleep, depression \& anxiety. SleepFoundation.org [Updated 18 September 2020, retrieved in 27 September 2020]; https://www.sleepfoundation.org/ excessive-sleepiness/health-impact/complex-relationshipbetween-sleep-depression-anxiety.

Vaughn CB, Freudenheim JL, Nie J, et al (2018). Sleep and breast cancer in the Western New York Exposures and Breast Cancer (WEB) study. J Clin Sleep Med, 14, 81-6.

Vin-Raviv N, Akinyemiju TF, Galea S, Bovbjerg DH (2018). Sleep disorder diagnoses and clinical outcomes among hospitalized breast cancer patients: a nationwide inpatient sample study. SCC, 26, 1833-40.

Wang YH, Li JQ, Shi JF, et al (2020). Depression and anxiety in relation to cancer incidence and mortality: a systematic review and meta-analysis of cohort studies. Mol Psychiatry, 25, 1487-99.

Wei M, Guo L, Zhu Y, et al (2019). Type C personality and depression among newly diagnosed breast cancer patients: the mediating role of sense of coherence. Neuropsychiatr Dis Treat, 15, 3519-29.

World Health Organization. Breast cancer. [Updated 8 May 2019, retrieved in 15 September 2020]; https://www.who. int/cancer/prevention/ diagnosis-screening/breast-cancer.

Zhang C, Hu G, Biskup E, et al (2018). Depression induced by total mastectomy, breast conserving surgery and breast reconstruction: A systematic review and meta-analysis. World J Surg, 42, 2076-85.

Zwielewski G, Sant' Ana V (2019). Mourning protocol details and the cognitive behavior therapy applicability. Int $J$ Soc Sci Humanit Invent, 6, 5417-23.

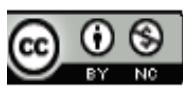

This work is licensed under a Creative Commons AttributionNon Commercial 4.0 International License. 